\title{
Study on the Deformation of Large Ring Radial-Axial Rolling Based on the FE Simulation of the Process
}

\author{
GU Rui-jie ${ }^{1,2,3}$, ZHANG Shu-lian ${ }^{1,2}$, YANG Da-xiang ${ }^{1,2}$, ZHENG Wen-da ${ }^{1,2,}$ JING Yun-hai ${ }^{1,2}$ and GU Ya-jie $^{4, *}$ \\ ${ }^{1}$ China National Heavy Machinery Research Institute Co. Ltd, Xi'an 710032, China \\ ${ }^{2}$ State Key Laboratory of Metal Extrusion and Forging Equipment Technology, Xi'an 710032, China \\ ${ }^{3}$ College of Materials Science and Engineering, Northwestern Polytechnical University, Xi'an 710072, China \\ ${ }^{4}$ Qinyang Vocational Secondary School, Qinyang 454550, China
}

\begin{abstract}
Ring radial-axial rolling technology is the best method to produce high quality large seamless ring. The reliable finite element model of large ring radial-axial rolling process is established. Based on the computing result of the FE model, the deformation of large ring radial-axial rolling process is studied. And it is found that the whole deformation feature of the ring depends on the material deformation of radial rolling area and axial rolling area, which is very useful to study deeply the forming process of large ring radial-axial rolling.
\end{abstract}

\section{Introduction}

Ring radial-axial rolling (Fig.1) is the continuous local forming process of ring diameter increasing through the continuous local pressing down on ring thickness and height of rollers ${ }^{[1,2]}$. The formed ring precision by radialaxial rolling is high, so the machinery allowance is small and the material utilization is high. The metal fiber structure by radial-axial rolling can improve the mechanical performance of formed ring. The action force of ring radial-axial rolling is much smaller than that of ring whole forging, but the production efficiency of ring radial-axial rolling is much higher than that of ring whole forging. So the technology of ring radial-axial rolling has been the best method to produce high quality large seamless ring ${ }^{[3-4] \text {. }}$

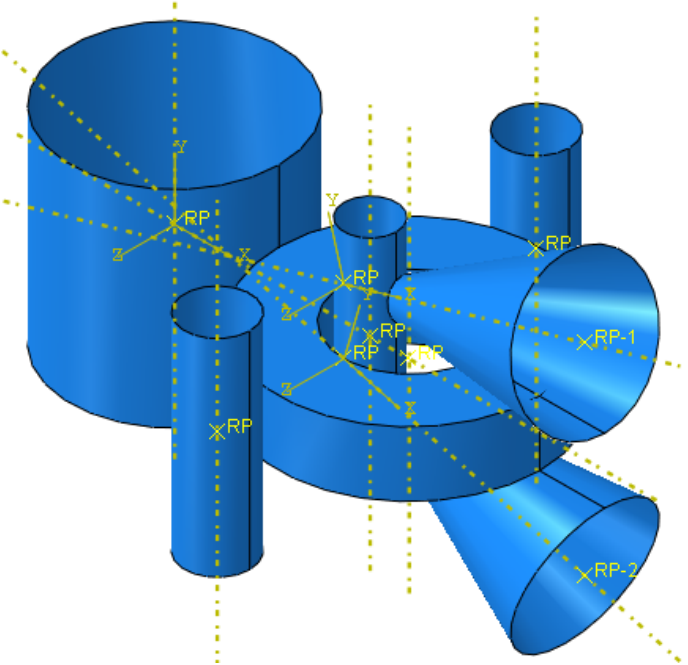

Fig. 1 Sketch of ring radial-axial rolling

The radial-axial rolling process of large ring is a complicated multiple nonlinear process of geometry, material and boundary under the coupling action of multiple fields and multiple factors, so it is difficult to study deeply the process. Some researches on the process of ring rolling have been done, but the research on the process of large ring radial-axial rolling ${ }^{[5-8]}$ is few and is not much satisfied with the factual production.

The reliable finite element model of large ring radialaxial rolling process is established in the study. With the model, the process is computed and analyzed, some basic deformation rule of the process is revealed, which is very useful to study deeply the forming process of large ring radial-axial rolling.

\section{FE model establishment of large ring radial-axial rolling process}

The finite element model of large ring radial-axial rolling process is established on the software platform of ABAQUS in the study. The main work includes the establishment of geometry model, the definition of material property, the application of dynamic explicit algorithm, the setting of contact condition, the meshing of deformation part, the application of load.

During the process of large ring radial-axial rolling, the deformation of rollers is much smaller than that of ring, so the rollers are treated as rigid parts and the ring treated as deformation part in the FE model. The ring material is defined with elastic-plastic constitutive equation. The dynamic explicit algorithm of ABAQUS/Explicit is used to compute the process of large ring radial-axial rolling and the technology of mass scale used to improve the computing efficiency. The contact conditions include the contact of ring and main roller, that of ring and mandrel, that of ring and cone roller, that of ring and two centering rollers. The hexahedron 8 node thermal-force coupling element of first order reduced integration is used to mesh the ring and the ALE technology used to control the mesh distortion.

The loads of all rollers are applied through velocity, angular velocity or displacement condition. Main roller rotates around its center axis with certain angular velocity and mandrel moves towards main roller according to the preset feeding curve. The up cone roller rotates around its center axis and the angular velocity of cone roller is matching with that of main roller. The ring rotating velocity driven by the main roller and that driven by the up cone roller are same. Meanwhile, the up cone roller moves towards the down cone roller according to the preset feeding curve. The backward travel of up cone roller and down cone roller is applied through displacement and the displacement value is the same as 
the increasing value of ring diameter. The load of centering rollers is applied through plane displacement and the location of centering rollers can be confirmed with ring diameter and the structure of the centering device.

\section{Symmetry of radial-axial rolling ring and improvement of its FE model}

With the FE model of large ring radial-axial rolling process, the effective stress and the effective strain of radial-axial rolling ring can be gained as Fig.2 and Fig. 3. It can be found that the stress and the strain of rolling ring are up-down symmetric. During the process of ring radial-axial rolling, axial rolling force and moment are applied through the pressing down and rotating of up cone roller, but the up cone roller and the down cone roller are up-down symmetric with regard to the middle section of rolling ring, so the deformation of rolling ring is also up-down symmetric.

According to the up-down symmetric deformation feature of rolling ring, the FE model of large ring radialaxial rolling process can be improved. In the FE model, the up half of ring blank is used and the symmetric constrain applied on the middle section of the ring blank. The down cone roller can be eliminated and the feeding value of up cone roller is only the half of real feeding value. The improved FE model of large ring radial-axial rolling process is shown as Fig.4 and its computing efficiency is twice of that of initial model.

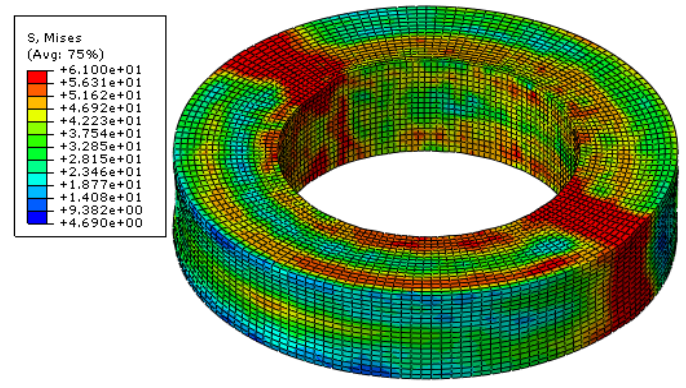

Fig. 2 Effective stress of radial-axial rolling ring

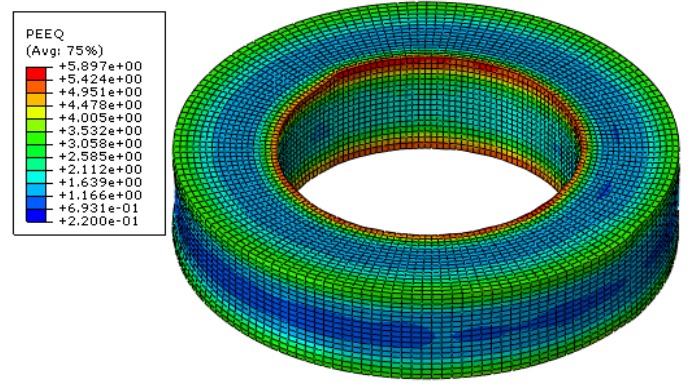

Fig. 3 Effective strain of radial-axial rolling ring

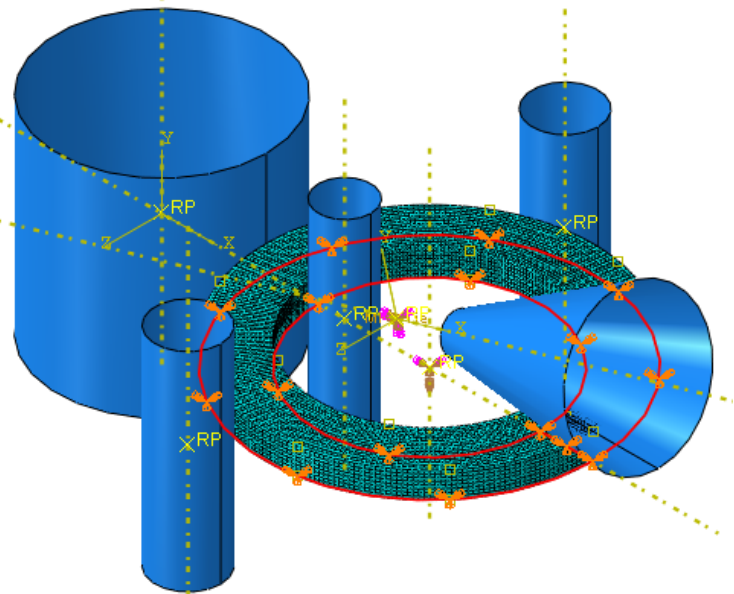

Fig.4 FE model of radial-axial rolling ring

\section{Reliability of the FE model of radial-axial rolling ring process}

The reliability of the FE model of radial-axial rolling ring process is validated through experiment. The experiment equipment is the large ring radial-axial rolling mill developed by the authors (Fig.5). The Main technique parameters of the mill are shown in Table 1.

Table 1. Main technique parameters

\begin{tabular}{|c|c|}
\hline Technique parameters & Value \\
\hline Main roller diameter & $1600 \mathrm{~mm}$ \\
\hline Mandrel diameter & $400 \mathrm{~mm}$ \\
\hline Cone roller angle & $32^{\circ}$ \\
\hline Cone roller available length & $1300 \mathrm{~mm}$ \\
\hline Main roller rotating angluar velocity & $1-2 \mathrm{rad} / \mathrm{s}$ \\
\hline Mandrel maximum feeding velocity & $2 \mathrm{~mm} / \mathrm{s}$ \\
\hline Up cone roller maximum feeding velocity & $2 \mathrm{~mm} / \mathrm{s}$ \\
\hline Ring outer diameter & $2000-8000 \mathrm{~mm}$ \\
\hline Ring thickness & $100-1000 \mathrm{~mm}$ \\
\hline Ring height & $100-1000 \mathrm{~mm}$ \\
\hline
\end{tabular}

The compare of FE computing result and experiment result of ring outer diameter is shown as Fig.6. It can be found that the FE computing result and the experiment result of ring outer diameter are almost same, the maximum error between them is smaller than $10 \%$. So the FE model of large ring radial-axial rolling process is reliable. 


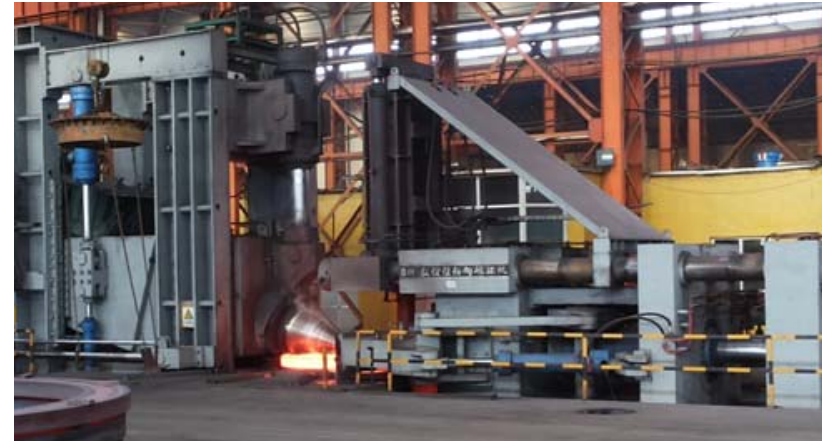

Fig. 5 Experiment mill of large ring radial-axial rolling

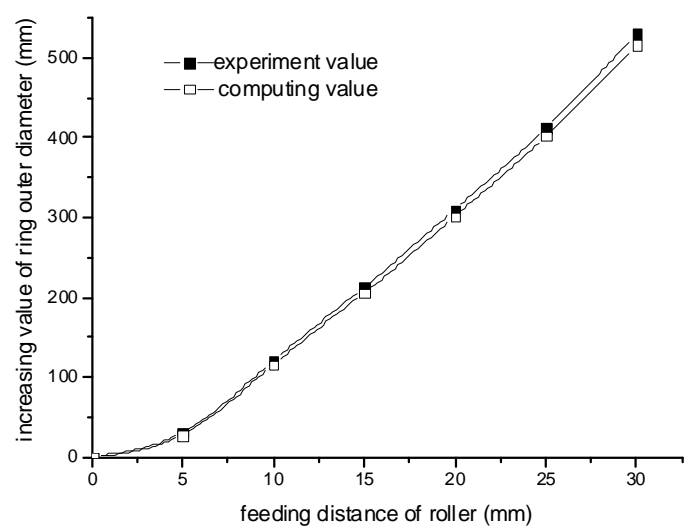

Fig. 6 Simulation value and experiment value of ring diameter increasing

\section{Deformation analysis of large ring radial-axial rolling process}

With the above FE model, the process of large ring radial-axial rolling is computed, the stress and strain developing rule can be gained. The effective stress and the stress of three cylindrical coordinate directions are shown as Fig.7. The effective strain and the strain of three cylindrical coordinate directions are shown as Fig.8.

Through analyzing the developing rule of the stress and strain during radial-axial rolling ring, it can be found that the ring thickness is decreased under big pressure stress when the material is rotated through the radial rolling deformation area, then the big pressure stress of thickness direction disappears, but the deformation created in the radial rolling deformation area keeps almost invariable before the material is rotated into the axial rolling deformation area. The ring height is decreased under big pressure stress when the material is rotated through the axial rolling deformation area, then the big pressure stress of height direction disappears, but the deformation created in the axial rolling deformation area keeps almost invariable before the material is rotated into the radial rolling deformation area. So the deformation of radial-axial rolling ring is created in the radial rolling deformation area and the axial rolling deformation area, the whole deformation feature of the ring depends on the material deformation of radial rolling area and axial rolling area.
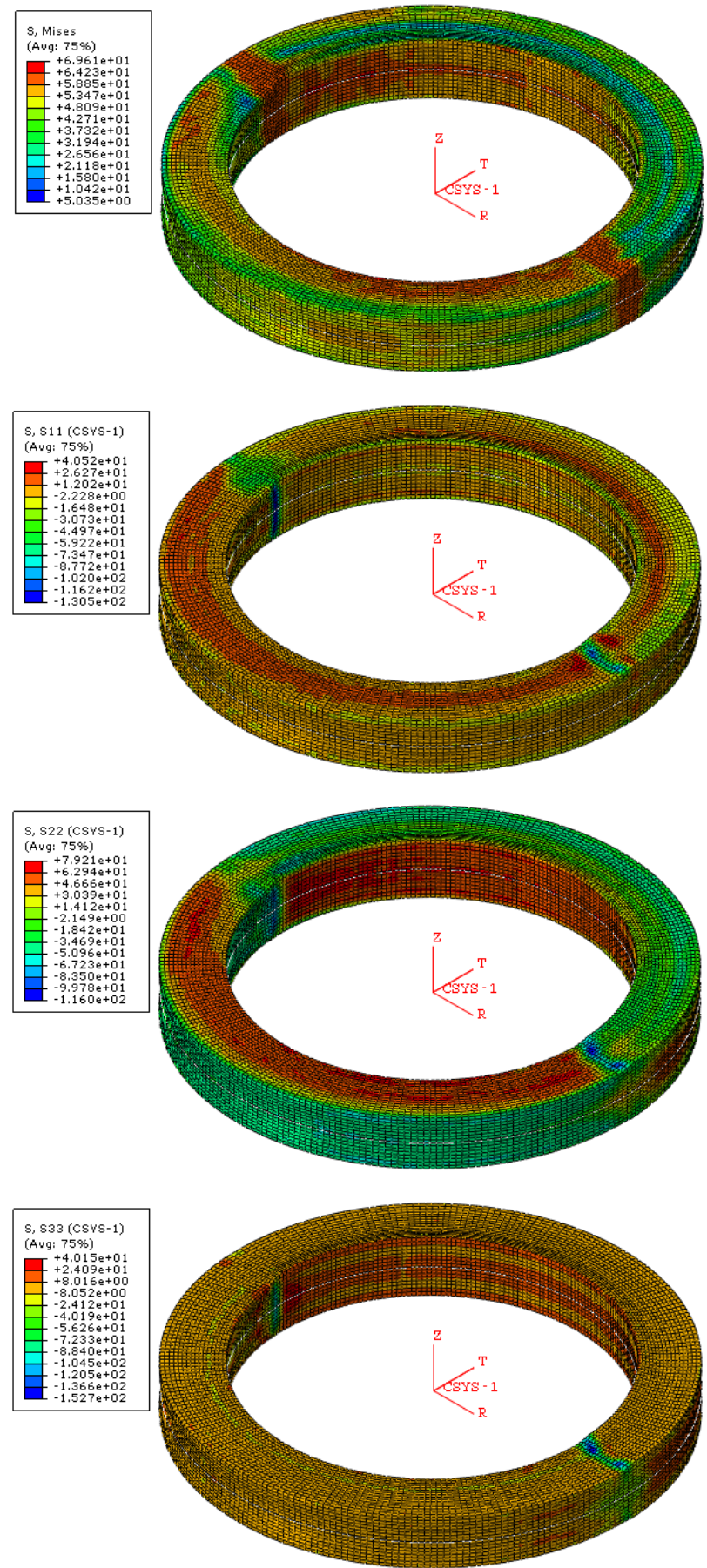

Fig. 7 Stress of radial-axial rolling ring

\section{Conclusion}

The finite element model of large ring radial-axial rolling process is established on the software platform of ABAQUS in the study. Based on the deformation symmetry of large ring radial-axial rolling process, the FE model is improved. Then the reliability of the FE model is validated through experiment. Based on the computing result of the FE model, the deformation rule of large ring radial-axial rolling process is studied. It is found that the deformation of radial-axial rolling ring is created in the radial rolling deformation area and the axial rolling deformation area, the whole deformation 
feature of the ring depends on the material deformation of radial rolling area and axial rolling area.
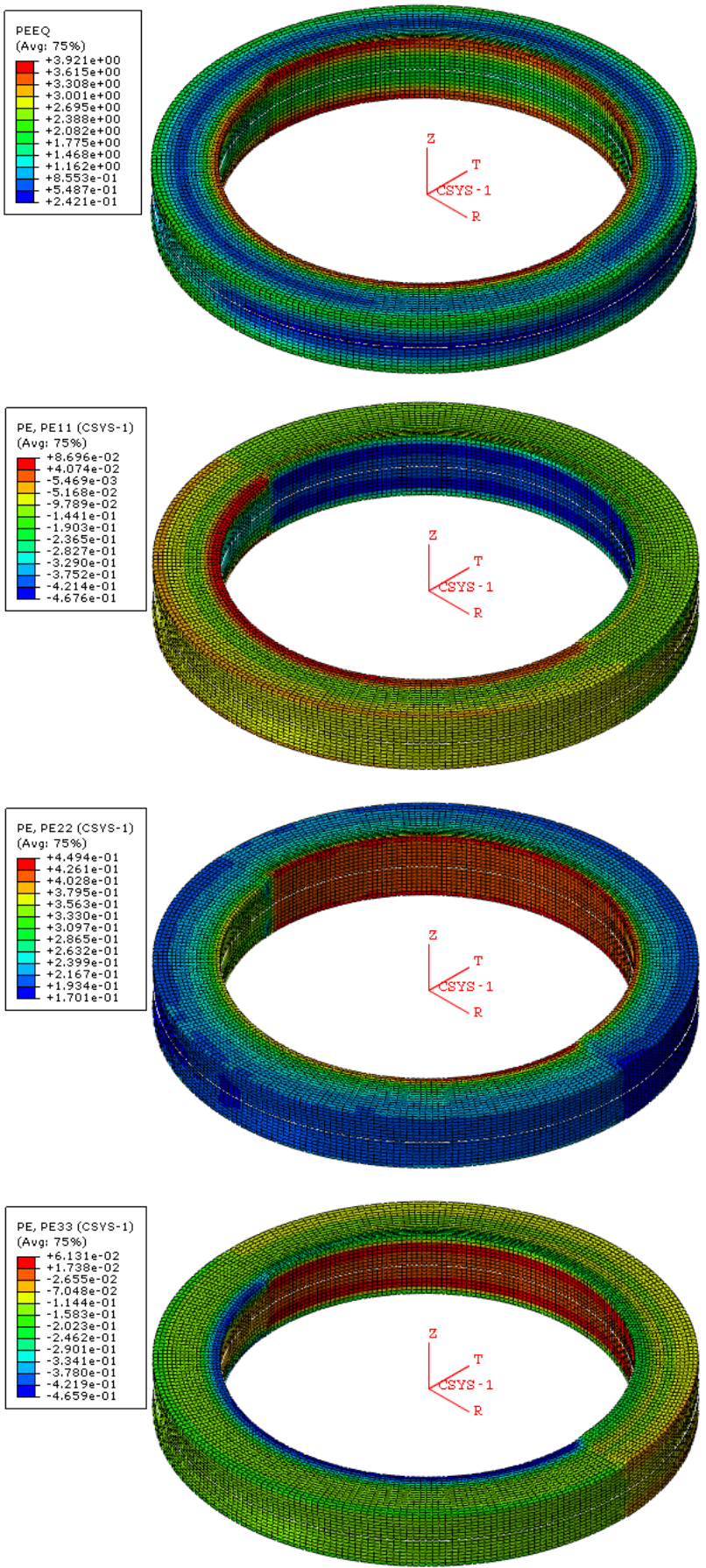

Fig. 8 Strain of radial-axial rolling ring

\section{Acknowledgement}

The authors would like to thank the National Science and Technology Major Project of China (No. 2010ZX04004131) and the National Program on Key Basic Research Project of China (No. 2012CB724300) for the supports given to this research.

\section{References}

[1] Lianggang Guo, He Yang. Towards a steady forming condition for radial-axial ring rolling [J]. International Journal of Mechanical Sciences, 53 (2011): 286-299

[2] J.L. Songa, A.L. Dowsona, M.H. Jacobsa, J. Brooksb, I. Beden. Coupled thermo-mechanical finite-element modeling of hot ring rolling process [J]. Journal of Materials Processing Technology, 121 (2002): 332340

[3] GU Rui-jie, ZHANG Shu-1ian, YANG Da-xiang, GAO Hong-zhang, XIN Hong-bin, CHENG Xiao-1e. Mechanics analysis on the moving beam of extra large ring radial rolling equipment and its structure optimization [J]. Heavy machinery, 2012(3): 137140 (in Chinese)

[4] GU Rui-jie, Quan Xiao-hui, ZHANG Shu-1ian, YANG Da-xiang, YANG Zhan-fei, LIANG Jian-zhu, SU Zhen-hua. Research on the guide rollers automatic location control of ring radial-axial rolling NC equipment [J]. Heavy machinery, 2013(6): 6-9 (in Chinese)

[5] Shuai Zhu, He Yang, Lianggang Guo, Linlin Hu, Xiaoqing Chen. Research on the effects of coordinate deformation on radial-axial ring rolling process by FE simulation based on in-process control [J]. International Journal of Advanced Manufacture Technology, 72 (2014): 57-68

[6] L. Hua, X.G. Huang, C.D. Zhu. Ring Rolling Theory and Technology. Mechanical Industry Press, Beijing, 2001. (in Chinese)

[7] Hua Lin, Pan Libo, Lan Jian, Qian Dongsheng. Simulation Research on Radial-axial Large Ring Rolling Technology. Chinese Mechanical Engineering, 2006, 17(19): 2020-2023 (in Chinese)

[8] Liu Dong, Wan Ziyong, Fu Mingjie, Zhang Hua. Kinematics Analysis in Radial/axial Ring Rolling. Chinese Mechanical Engineering, 2008, 19(4): 12761280 (in Chinese) 